\title{
Respiratory and Gastrointestinal Management of an Infant with a Birth Weight of 258 Grams
}

\author{
Ryo Itoshima, MD ${ }^{1,2}$ Arata Oda, $\mathrm{MD}^{1}$ Ryo Ogawa, MD ${ }^{1}$ Toshimitsu Yanagisawa, $\mathrm{PhD}^{1}$ (1) \\ Takehiko Hiroma, $\mathrm{PhD}^{1}$ Tomohiko Nakamura, $\mathrm{PhD}^{1}$
}

1 Division of Neonatology, Nagano Children's Hospital, Nagano, Japan

2 Department of Pediatrics, University of Turku, Turku, Finland

AJP Rep 2022;12:e89-e95.
Address for correspondence Ryo Itoshima, MD, Division of Neonatology, Nagano Children's Hospital, 3100, Toyoshina, Azumino City, Nagano - 399-8288, Japan; Department of Pediatrics, University of Turku, Fl-20014 Turun yliopisto, 20500 Turku, Finland; and Division of Neonatology, Turku University Hospital, Kiinamyllynkatu 4-8, 20521 Turku, Finland

(e-mail: ryitos@utu.fi; itoshima.ryo@gmail.com).

\author{
Abstract \\ Keywords \\ - tiny infant \\ - extremely low birth \\ weight \\ - small for gestational \\ age \\ - high-frequency \\ oscillatory ventilation \\ with volume \\ guarantee \\ - noninvasive neurally \\ adjusted ventilatory \\ assist \\ - meconium-related \\ ileus \\ - transpyloric tube \\ feeding \\ - cow milk-based \\ fortifier \\ - medium-chain \\ triglyceride oil
}

Today, more infants weighing less than or equal to $300 \mathrm{~g}$ are born, and they survive because of the improvements in neonatal care and treatment. However, their detailed clinical course and neonatal intensive care unit management remain unknown due to their low survival rate and dearth of reports. A male infant was born at 24 weeks and 5 days of gestation and weighed $258 \mathrm{~g}$. The infant received 72 days of invasive and 92 days of noninvasive respiratory support, including high-frequency oscillatory ventilation with volume guarantee and noninvasive neurally adjusted ventilatory assist. Meconium-related ileus was safely treated using diatrizoate. Although the infant was diagnosed with severe bronchopulmonary dysplasia and retinopathy of prematurity requiring laser photocoagulation, he had no other severe complications. He was discharged 201 days postdelivery (3 months of corrected age) with a weight of $3.396 \mathrm{~kg}$. Although managing infants weighing less than or equal to $300 \mathrm{~g}$ is difficult, our experience shows that it is possible by combining traditional and modern management methods. The management of such infants requires an understanding of the expected difficulties and adaptation of existing methods to their management. The management techniques described here should help improve their survival and long-term prognosis. received

September 20, 2021 accepted after revision October 20, 2021
DOI https://doi.org/ 10.1055/a-1678-3755. ISSN 2157-6998.
(C) 2022. The Author(s).

This is an open access article published by Thieme under the terms of the Creative Commons Attribution-NonDerivative-NonCommercial-License, permitting copying and reproduction so long as the original work is given appropriate credit. Contents may not be used for commercial purposes, or adapted, remixed, transformed or built upon. (https://creativecommons.org/ licenses/by-nc-nd/4.0/)

Thieme Medical Publishers, Inc., 333 Seventh Avenue, 18th Floor, New York, NY 10001, USA 
Today, more infants weighing less than or equal to $500 \mathrm{~g}$ are being managed, ${ }^{1}$ and although a lower birth weight is associated with a higher neonatal mortality rate, ${ }^{2}$ the survival rate among these low-birth-weight infants has improved. ${ }^{3}$ Thus, more infants weighing less than or equal to $300 \mathrm{~g}$ can survive at discharge or longer. Nevertheless, their survival rate is still very low. Previous regional and national cohort studies have shown that the survival rates of infants weighing less than or equal to $500 \mathrm{~g}$ in neonatal intensive care units (NICUs) range from 12 to $55 \%{ }^{3-7}$ However, for infants weighing less than or equal to $300 \mathrm{~g}$, the survival rate is much lower at 4 to $18 \%{ }^{3,5}$ Even in recent years, greater than $50 \%$ of infants weighing less than or equal to $400 \mathrm{~g}$ did not receive active treatment at birth. ${ }^{8}$ As a result, only 42 infants weighing less than $300 \mathrm{~g}$ have been registered in the database of the University of Iowa, which is the international database for infants weighing less than 400 g. ${ }^{9}$ Additionally, the development of infants weighing less than or equal to $300 \mathrm{~g}$ has only been mentioned in two articles. Two of the three infants weighing less than or equal to $300 \mathrm{~g}$ included in a Japanese cohort study between 2003 and 2012 were diagnosed with neurodevelopmental impairment at the age of 3 years. ${ }^{10}$ Besides, a report of two cases described that one of the two infants was diagnosed with neurodevelopmental impairment at the age of 2 years. ${ }^{11}$ Due to the low survival rate of these infants, limited data are available on infants weighing less than or equal to $300 \mathrm{~g}$, especially about their clinical courses. Moreover, data on their clinical management at neonatal resuscitation or in the NICU to ensure survival remain limited. Thus, even if clinicians are aware of an effective management technique for infants weighing greater than $300 \mathrm{~g}$, they may hesitate in terms of adapting it for infants weighing less than or equal to $300 \mathrm{~g}$ because of the lack of available evidence or experience.

In this case report, we describe our experience in managing a very small male infant with a birth weight of $258 \mathrm{~g}$, who is still alive and healthy at 2 years of corrected age. Particular emphasis is placed on the respiratory and gastrointestinal management in the NICU. We have also described the routine management of very preterm and/or extremely low-birthweight infants in our NICU and have discussed how we adapted these management techniques to ensure survival of this infant.

\section{Case Presentation}

\section{Pregnancy}

A very preterm male infant was born to a G4P3 Japanese mother. Her blood pressure before pregnancy was mildly elevated, and it was exacerbated after pregnancy. In addition, fetal growth restriction was recognized. She was referred and admitted to our hospital. A nonreassuring fetal status was determined, and hence, the decision was made to deliver the infant via emergency cesarean section at 24 weeks and 5 days of gestation. Antenatal corticosteroids had already been administered 1 and 2 days before the delivery. Active treatment of the infant was decided based on the gestational age and the fetal condition. The infant's parents were kept fully informed prenatally, and their consent was obtained. The umbilical arterial $\mathrm{pH}$ was 7.335 , and the weight of the placenta was
120 g. Pathological analysis of the placenta revealed only ischemic changes and no signs of chorioamnionitis.

\section{Neonatal Resuscitation}

At our hospital, neonatal resuscitation for very preterm infants is always conducted in closed incubators to prevent hypothermia and avoid stress on the infants. The incubator was preheated to $39^{\circ} \mathrm{C}$, and the humidity was maintained at $95 \%$. Bag-mask ventilation was immediately started with Infant Silicone Mask 0/0 (Laerdal Medical AS, Stavanger, Norway) after the infant arrived at the incubator. Simultaneously, he was covered with a plastic wrap after wiping off the amniotic fluid. After a few failed attempts by several skilled neonatologists, he was successfully orally intubated with a $2.0-\mathrm{mm}$ endotracheal tube using a size-00 laryngoscope blade and a stylet at 16 minutes of life. The Apgar score was 2, 4, and 6 at 1, 5, and 10 minutes, respectively. $\mathrm{SpO}_{2}$ was approximately $90 \%$ even after the oxygen concentration was raised from 30 to $100 \%$, and air entry was poor even after intubation. Therefore, $45 \mathrm{mg}(100 \mathrm{mg} / \mathrm{kg}$ calculated by the estimated prenatal weight) of surfactant was administered in three divided doses according to his position at 30 minutes in the resuscitation room. When he was transferred to the NICU at 53 minutes, his vital signs were as follows: temperature, $35.6^{\circ} \mathrm{C}$; heart rate, $160 \mathrm{bpm}$; and $\mathrm{SpO}_{2}, 95 \%$ in $80 \%$ of oxygen. At admission, his body weight was $258 \mathrm{~g}(-4.95$ standard deviation score [SDS]). To avoid unnecessary irritation, other physical measurements were taken 8 days after delivery; his length was $22.0 \mathrm{~cm}$ (-3.75 SDS), and his head circumference was $18.3 \mathrm{~cm}$ (-2.57 SDS). He was diagnosed as being small for gestational age (SGA), and his appearance at 1 day of age is shown in - Fig. 1.

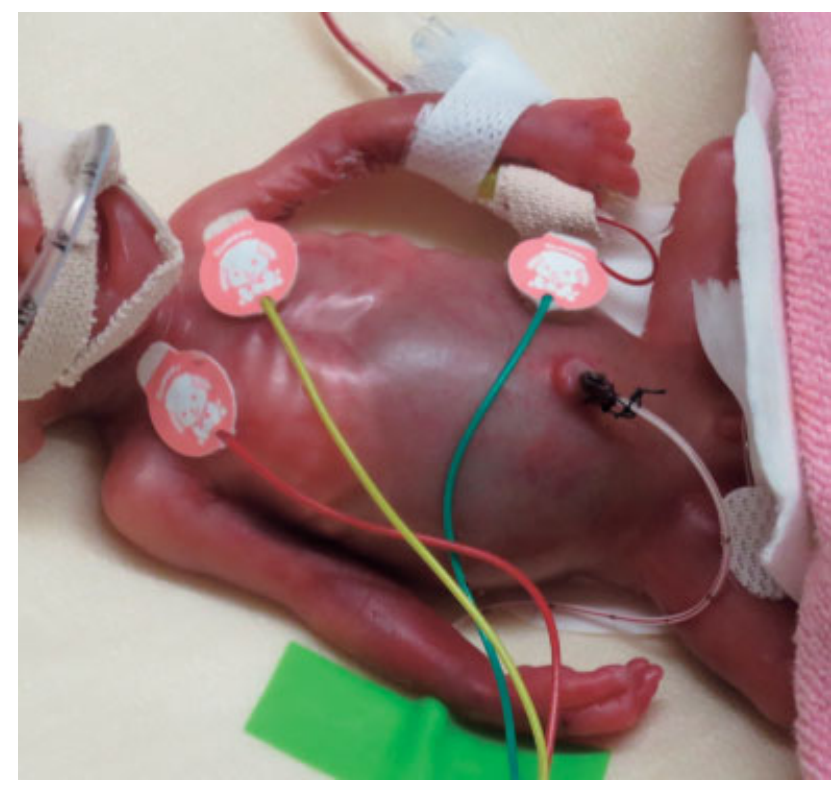

Fig. 1 The appearance of the infant at 1 day of age. He was lying on the decompression mat and his skin was already maturing. He was intubated with a $2.0-\mathrm{mm}$ endotracheal tube. A 27-gauge double-lumen peripherally inserted central catheter was placed through the left vena basilica near the axilla. A 5-Fr umbilical venous catheter was also placed. A 4-Fr orogastric tube and urethral catheter were placed. The abdomen was distended and purple due to meconium-related ileus. 


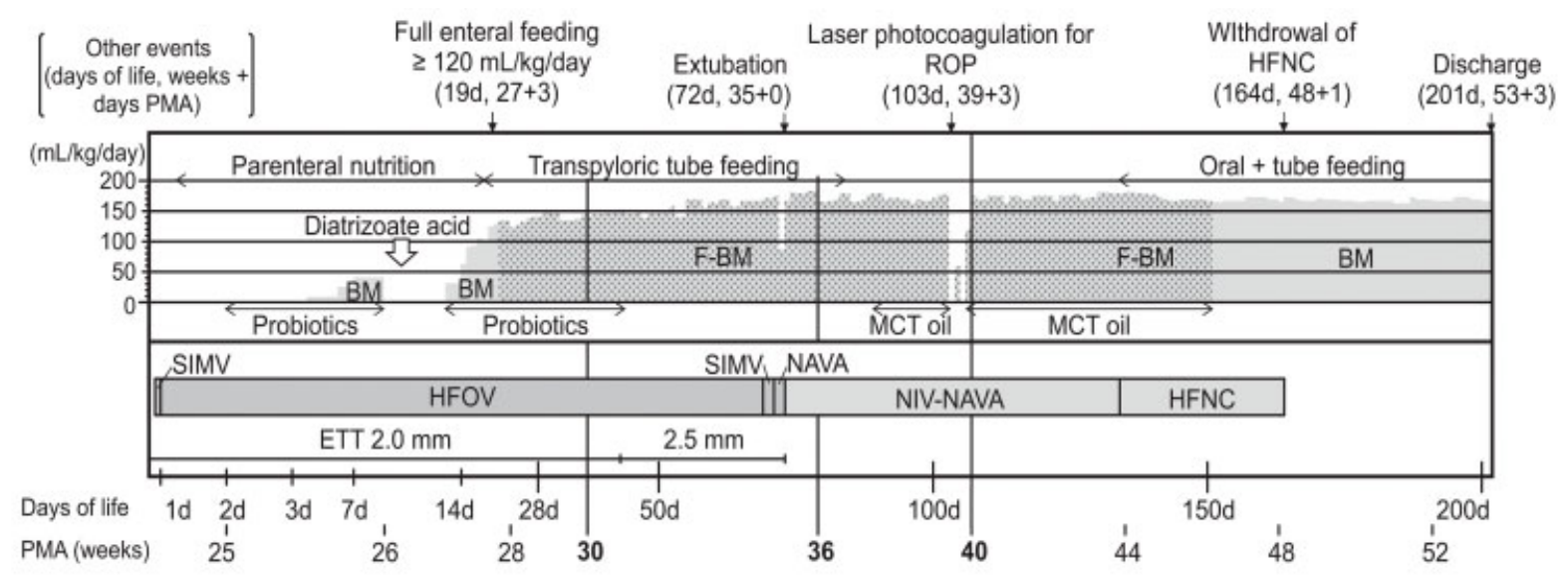

Fig. 2 The summary of the clinical course in the neonatal intensive care unit. The upper graph shows the amount of feeding ( $\mathrm{mL} / \mathrm{kg} / \mathrm{d})$, nutrition administration methods, and gastrointestinal events. The lower graph shows the types of ventilatory support and the size of the ETT (BM, mother's breast milk; ETT, endotracheal tube; F-BM, fortified breast milk; HFNC, high-flow nasal cannula; HFOV, high-frequency oscillatory ventilation; MCT, medium-chain triglyceride; NAVA, neurally adjusted ventilatory assist; NIV-NAVA, noninvasive ventilation neurally adjusted ventilatory assist; PMA, postmenstrual age; ROP, retinopathy of prematurity; SIMV, synchronized intermittent mandatory ventilation).

\section{Management in the NICU}

The detailed course of respiratory management is shown in - Fig. 2. Immediately after admission, respiratory management with synchronized intermittent mandatory ventilation (SIMV) was initiated, with a peak inspiratory pressure (PIP)/ positive end-expiratory pressure of $22 / 5 \mathrm{~cm} \mathrm{H}_{2} \mathrm{O}$, respiratory rate of $30 \mathrm{bpm}$, inspiratory time of 0.6 seconds, and flowtrigger sensitivity of $0.2 \mathrm{~L} / \mathrm{min}$. The first venous blood gas analysis revealed a $\mathrm{pH}$ of $7.212, \mathrm{pCO}_{2}$ of $57.6 \mathrm{~mm} \mathrm{Hg}$, and $\mathrm{HCO}_{3}{ }^{-}$of $22.3 \mathrm{mEq} / \mathrm{L}$. However, the oxygen demand increased and respiratory status deteriorated a few hours later. Therefore, SIMV was changed to high-frequency oscillatory ventilation (HFOV), which successfully improved the respiratory condition. The initial mean airway pressure was $11 \mathrm{~cm} \mathrm{H}_{2} \mathrm{O}$, and it was maintained at $8 \mathrm{~cm} \mathrm{H}_{2} \mathrm{O}$ after the acute phase. Four days after delivery, as his respiratory condition stabilized, respiratory management using HFOV with volume guarantee (HFOV-VG) was started with a high-frequency tidal volume (VThf) of $0.7 \mathrm{~mL}(2.7 \mathrm{~mL} / \mathrm{kg})$. The VThf was adjusted based on blood gas analysis, and hence, it varied from 0.7 to $1.0 \mathrm{~mL}$. HFOV-VG was continued until 50 days of life. All HFOV management was conducted using Babylog VN500 (Dräger, Lübeck, Germany).

His respiratory condition worsened again 2 weeks after delivery. The transpyloric tube feeding was started to prevent silent aspiration. In addition, the endotracheal tube was changed from 2.0 to $2.5 \mathrm{~mm}$ at 30 weeks postmenstrual age (PMA) and a weight of $472 \mathrm{~g}$. Two courses of hydrocortisone were also administered to improve the respiratory status. The oxygen demand slowly decreased, and he was extubated at 35 weeks PMA and a weight of $864 \mathrm{~g}$. In our hospital, extremely preterm infants are usually extubated at 30 weeks PMA or a weight of $1 \mathrm{~kg}$ based on the general condition; however, this infant was too small at 30 weeks PMA. Before extubation, HFOV was replaced by SIMV and then neurally adjusted ventilatory assist (NAVA) according to our routine. After extubation, noninvasive NAVA
(NIV-NAVA) was required to avoid reintubation. The initial NAVA level was $1.8 \mathrm{~cm} \mathrm{H}_{2} \mathrm{O} / \mu \mathrm{V}$. Because of the high electrical activity of the diaphragm (Edi) of 15 to $25 \mu \mathrm{V}$ and poor weight gain, the NAVA level was raised from 1.5 to $1.8 \mathrm{~cm} \mathrm{H}_{2} \mathrm{O} / \mu \mathrm{V}$. A few trials of NIV-NAVA withdrawal were conducted, but these failed because of the exacerbation of oxygen demand, respiratory effort, or mild pulmonary hypertension. After 2 months, the NIV-NAVA support was replaced with a highflow nasal cannula at 43 weeks PMA. The use of the high-flow nasal cannula was finally discontinued at 48 weeks PMA (164 days), and no more respiratory support, including oxygen supply, was required. Echocardiography performed at discharge did not show any sign of pulmonary hypertension or ductus arteriosus. He received a diagnosis of severe bronchopulmonary dysplasia (BPD) at 36 weeks PMA, as defined by the National Institute of Child Health and Human Development. ${ }^{12}$ However, no respiratory support was required at discharge.

The very thin and fragile umbilical vessels of the infant made insertion of the umbilical catheter at admission difficult. Only one 3.5-Fr catheter could be finally inserted, which was initially thought to be an arterial line but proved to be a venous line a few days later. Thereafter, we peripherally inserted a central catheter during normal saline bolus infusion from the umbilical catheter. The vena basilica near the axilla was punctured with a 24 -gauge indwelling needle, and a 27-gauge double-lumen peripherally inserted central catheter was placed. Because the infant had hypotension, dopamine, dobutamine, and a total of $3 \mathrm{mg} / \mathrm{kg}$ of intravenous hydrocortisone were administered in the first 48 hours of life. The target pressure was set at $24 \mathrm{~mm} \mathrm{Hg}$ of mean blood pressure because the target pressure is usually defined as the same value as the infant's gestational week. Additionally, echocardiography revealed hypovolemia and pulmonary hypertension, which were reasons for treating the hypotension. Nitric oxide was also administered to deal with mild pulmonary hypertension until 36 hours of life. The systemic 
hypotension and pulmonary hypertension improved within the first 8 hours. The total fluid volume in the first 24 hours was $167 \mathrm{~mL} / \mathrm{kg} / \mathrm{d}$. The ductus arteriosus closed at 12 hours of life without any treatment. Otherwise, indomethacin would be administered prophylactically after the pulmonary hypertension improved or therapeutically when patent ductus arteriosus was proved to be symptomatic by echocardiography.

The detailed nutritional course is also shown in - Fig. 2. Parenteral nutrition was initiated at 0 days and continued until 18 days. Enteral nutrition was initiated at 2 days with his mother's breast milk at $0.5 \mathrm{~mL}$ three times a day through a 4-Fr orogastric tube. Administration of probiotics was also initiated the same day and continued until 1 month of age. The amount of enteral nutrition was gradually increased, but abdominal distention due to meconium-related ileus (MRI) worsened despite frequent glycerin enema. At 10 days, Gastrografin (diatrizoate meglumine and diatrizoate sodium; Bayer Yakuhin, Osaka, Japan) was administered rectally for therapeutic purposes while acquiring several abdominal radiographs in the NICU. A total of $8 \mathrm{~mL}$ of fourfold diluted diatrizoate (i.e., from 59.73 to $14.93 \mathrm{w} / \mathrm{v} \%$ ) was used. As diatrizoate did not reach the ileocecum, an additional $1 \mathrm{~mL}$ of four times diluted diatrizoate was administered later through the gastric tube. The MRI findings showed improvement after adequate defecation was achieved, and the amount of enteral nutrition was increased again. However, as his respiratory condition worsened, orogastric tube feeding was replaced with transpyloric tube feeding between 18 and 84 days. Fortified breast milk feeding was initiated at 21 days after the amount of enteral feeding reached $100 \mathrm{~mL} / \mathrm{kg} / \mathrm{d}$ and was continued until 5 months of life. Medium-chain triglyceride oil was also administered between 3 and 5 months of life to ensure weight gain. As a result, the calories were maintained at approximately $150 \mathrm{kcal} / \mathrm{kg} / \mathrm{d}$ with fortification only or approximately $175 \mathrm{kcal} / \mathrm{kg} / \mathrm{d}$ with fortification and medium-chain triglyceride oil. Oral feeding was started at 4 months of life ( 44 weeks PMA) after NIV-NAVA was replaced with a high-flow nasal cannula. However, fatigue prevented him from continuing to suckle. He still required tube feeding at discharge (53 weeks PMA). Almost all enteral nutrition was provided by his mother's breast milk.

Nursing care in the first 72 hours was provided based on minimal handling. Tracheal suctioning was conducted only when required. The infant's position was kept supine, with the head raised to prevent intraventricular hemorrhage. As this infant was diagnosed with SGA, glycerin enema and air release via the anal route were initiated within 48 hours. A decompression mat and skin protector were used to avoid skin trouble (-Fig. 1). At first, the environment inside the incubator was as hot and humid as in the resuscitation room. Then, the temperature and humidity were gradually lowered, while considering the body temperature, skin maturity, diuretic status, and electrolytes.

No abnormalities, including intraventricular hemorrhage or periventricular leukomalacia, were observed on magnetic resonance imaging performed at 49 weeks PMA. His hearing was also normal, as determined using the auditory steadystate response at 2 years of corrected age. No obvious infection was detected. He required laser photocoagulation for his retinopathy of prematurity, but the posttreatment course was good and no additional treatment was required.

He was discharged at 201 days (6 months of life or 3 months of corrected age) and continued tube feeding at home. His physical measurements at discharge were as follows: weight, $3.396 \mathrm{~kg}$ (-3.8 SDS); length, $47.0 \mathrm{~cm}$ (-6.5 SDS); and head circumference, $33.5 \mathrm{~cm}$ (SDS at corrected age).

\section{Discussion}

The clinical course of infants weighing less than or equal to $300 \mathrm{~g}$ remains unclear because of their low survival rate. Only four case reports showed the detailed clinical course of infants weighing less than or equal to $300 \mathrm{~g}$ in the NICU. ${ }^{13-16}$ In the present case, we encountered several difficulties rarely observed in the management of infants weighing greater than $500 \mathrm{~g}$. However, we could overcome these difficulties with minimal sequelae by applying relatively well-known methods for managing extremely low-birth-weight infants. The employed methods highlight the special considerations and solutions required in the management of infants weighing less than or equal to $300 \mathrm{~g}$. In this discussion, we consider the respiratory and gastrointestinal management techniques employed in this case from a clinical perspective because of the many associated difficulties.

First, intubation of infants weighing less than or equal to $300 \mathrm{~g}$ is very difficult. In this case, successful intubation took 9 minutes from the first attempt and required efforts from several skilled neonatologists. This experience highlights the importance of being well prepared before resuscitation and preparing the laryngoscope with the smallest blade (size-00 if available), a $2.0-\mathrm{mm}$ endotracheal tube, and stylet. We must also check beforehand if the stylet can be inserted into the $2.0-\mathrm{mm}$ endotracheal tube because stylet insertion is sometimes impossible. Confirmation of successful intubation is also difficult. Even with an end-tidal $\mathrm{CO}_{2}$ detector, it can be difficult to immediately determine successful intubation because of the small tidal volume. Therefore, the observation period should be sufficient, and a comprehensive judgment should be made using an end-tidal $\mathrm{CO}_{2}$ detector and other physical or vital signs to determine successful intubation.

The next difficulty we faced was with synchronizing breathing. In this case, it was initially impossible to synchronize breathing with the infant's respiration even with the lowest flow- or pressure-trigger sensitivity. This was possible because of his small tidal volume, which caused labored breathing and increased oxygen demand. As his blood pressure was too low to administer sedative drugs, SIMV was changed to HFOV, which worked well. Nevertheless, it should be noted that the respirator may not be able to detect the small tidal volume of such infants. HFOV can be an alternative solution for such infants weighing less than or equal to $300 \mathrm{~g}$, and it facilitated the long-term management in this case.

HFOV has been demonstrated to not only reduce lung injury in an animal model ${ }^{17}$ but also improve the long-term lung function of preterm infants in a clinical setting compared with 
conventional ventilation. ${ }^{18,19}$ In addition, HFOV-VG is becoming established as a new respiratory management technique for very preterm infants, and it has the potential to improve their respiratory prognosis further. During the HFOV-VG mode of Babylog VN500, the pressure amplitude is automatically adjusted to achieve the set VThf. Compared with HFOV without volume guarantee, HFOV-VG can achieve a more stable VThf, blood $\mathrm{CO}_{2}$ level, and $\mathrm{CO}_{2}$ diffusion coefficient. ${ }^{20,21}$ These benefits are important for infants weighing less than or equal to $300 \mathrm{~g}$ who require delicate management. HFOV-VG may also have the potential to reduce lung injury, which may reduce the incidence of $\mathrm{BPD}$ or improve the long-term respiratory prognosis. In our NICU, the HFOV-VG mode delivered using Babylog VN500 is often used for the acute and chronic respiratory management of very preterm infants. Presently, a recommended VThf to achieve normocapnia is not available. Hence, the initial setting of VThf should be 1.6 to $1.9 \mathrm{~mL} / \mathrm{kg}$, as recommended in previous reports, ${ }^{22,23}$ or should be determined using the value of VThf shortly before the change to HFOV-VG and adjusted according to the results of blood gas analysis. In this case, the infant was managed with HFOV-VG between 4 and 50 days. Blood samples were collected every 1 to 3 days, and the VThf was adjusted appropriately. A higher blood $\mathrm{CO}_{2}$ level of 45 to $60 \mathrm{~mm} \mathrm{Hg}$ is permitted in our management strategy to reduce lung injury. Although further studies on respiratory prognosis are required, HFOV-VG is a promising option for the respiratory management of infants weighing less than or equal to $300 \mathrm{~g}$ in both the acute and chronic phases.

NAVA is a new mode of respiratory support and is a mechanical ventilatory method for preterm infants. NAVA assists the patients' respiration based on their Edi signals sensed through a special catheter. Thus, not only the timings of breath initiation but also the PIP and inspiratory times are determined based on the infant's breathing. NAVA usually implies invasive respiratory management, but its noninvasive use is also possible by using nasal masks or prongs via a technique called NIV-NAVA. In our NICU, NIV-NAVA is the first choice for noninvasive respiratory support after extubation, especially for very preterm and/or extremely low-birth-weight infants. Compared with nasal continuous positive airway pressure, NIV-NAVA is more useful for successful extubation, especially in infants with more severe respiratory conditions. ${ }^{24-26}$ In addition, noninvasive ventilation can help avoid ventilator-associated pneumonia and airway and lung trauma, which are the drawbacks of invasive ventilator management. Although NAVA and NIV-NAVA have been used in smaller infants, this is one of the smallest infants to date whose respiration was supported over a long period by using NIV-NAVA. In this case, NIV-NAVA could support his lungs with severe BPD and mild pulmonary hypertension for 2 months without the need for intubation, which would risk further exacerbation of his BPD. NIV-NAVA is useful for ensuring successful extubation and long-term ventilatory support after extubation in infants with a birth weight of less than or equal to $300 \mathrm{~g}$. Nevertheless, further research is warranted on NIV-NAVA, especially on its effects on long-term respiratory prognosis.
The nutritional and gastrointestinal management in this case was also interesting. Since most infants weighing less than or equal to $500 \mathrm{~g}$ receive a diagnosis of SGA, ${ }^{3}$ they have a high risk of developing MRI, an obstruction of the meconium in the intestine caused by a difficulty in meconium excretion. The most effective treatment for MRI currently available is diatrizoate administered rectally or orally if required. ${ }^{27-30}$ Perforation, hypothermia, hypovolemia, and electrolyte loss are the complications of diatrizoate enema to be aware of. ${ }^{31}$ In this case, we followed our usual approach for infants suspected of having MRI: (1) administer a minimum amount of breast milk and increase the dose carefully; (2) increase the concentration and frequency of enemas; (3) apply continuous intragastric suction using an 8-Fr Salem Sump tube (Covidien Japan, Tokyo, Japan) to decompress the upper digestive tract; (4) administer diatrizoate rectally (or orally in addition if the former is ineffective) when digestive tract perforation is suspected; and (5) perform surgical treatment if required. Diatrizoate (originally $59.73 \mathrm{w} / \mathrm{v} \%$ ) is usually diluted three to five times with normal saline and administered by pediatric surgeons in small doses rectally under radiographic guidance. If the infant's clinical condition is stable, the infant is transferred out of the NICU and fluoroscopy is used during diatrizoate administration. If the infant's condition is not stable, abdominal radiography is performed repeatedly in the NICU during drug administration. The dosage of diatrizoate is determined based on the radiographic findings. Radiographs are also acquired after diatrizoate administration to assess treatment effectiveness. As in this case, several doses of diatrizoate or additional oral administrations are sometimes required. Prophylactic administration of diatrizoate has been reported to be effective against $\mathrm{MRI}^{32}$; however, it may increase the risk of aspiration pneumonia. Currently, no clear recommendations are available regarding when and how to administer diatrizoate in such cases.

In terms of preventing BPD, transpyloric tube feeding can be a viable option. Studies have hypothesized that preterm infants suffer from aspiration to a greater or lesser degree depending on gastroesophageal reflux, which is also related to the onset and progression of BPD. ${ }^{33}$ As transpyloric tube feeding is effective in reducing the symptoms of gastroesophageal reflux, ${ }^{34,35}$ it may also be effective in preventing BPD caused by microaspiration. However, its effectiveness and safety in preterm infants remain unclear. ${ }^{36}$ In our unit, transpyloric tube feeding is initiated when a preterm infant shows any clinical sign of aspiration, for example, bradycardia and/or hypoxia during feeding or suspicion of breast milk in the tracheal aspirate. We use a 5-Fr and 80-cm Kangaroo New Enteral Feeding Tube (Covidien Japan, Tokyo, Japan) as the transpyloric tube for preterm infants. It is inserted blindly by neonatologists, and then radiography is performed to detect whether its tip is beyond the pylorus. The insertion and management can be performed safely by skilled neonatologists and nurses. In this case, a 4-Fr normal feeding tube was used as the transpyloric tube instead until the infant had gained sufficient physique because a $5-\mathrm{Fr}$ tube seemed too thick and dangerous to be inserted and placed in this tiny 
infant. Transpyloric tube feeding can be an alternative feeding method even for infants weighing less than or equal to $300 \mathrm{~g}$ and could prevent microaspiration and, perhaps, even BPD.

Promoting growth in preterm infants, especially in the NICU, is important for their long-term neurodevelopmental and growth prognosis. ${ }^{37-40}$ The most common nutritional method used to enhance growth in the NICU is a cow milkbased fortifier. ${ }^{41}$ One of the alternatives is the addition of medium-chain triglyceride oil, ${ }^{42,43}$ which is used in our NICU at a dose of 1 to $3 \mathrm{~g} / \mathrm{kg} / \mathrm{d}$ for infants with poor weight gain. In this case, since the infant's mother provided enough breast milk, a cow milk-based fortifier was used as in normal preterm infant management. However, the growth rate was still insufficient after extubation; no weight gain was observed in the first week. Therefore, the NAVA level was increased to reduce energy consumption, and medium-chain triglyceride oil was added to the enteral nutrition. Consequently, the growth rate improved, and the SDS of his weight at discharge improved from that at birth.

The limitation of this report is that it is a single case report. The management techniques applied in this case may not apply to other infants weighing less than or equal to $300 \mathrm{~g}$. It may be possible to standardize the management of infants weighing less than or equal to $300 \mathrm{~g}$ if more cases are reported in the future. Nevertheless, it is important to recognize in advance that clinical problems such as those observed in this case may also be observed in other similar cases. In addition, although infants weighing less than or equal to $300 \mathrm{~g}$ require very delicate management, the clinical problems can be overcome by applying conventional treatments commonly used at each institution. We conclude that one of the main reasons this male infant with a birth weight of $258 \mathrm{~g}$ could survive is that we successfully dealt with many clinical difficulties using both traditional and modern management methods commonly used in our NICU. The strength of this article is that it is one of only a few reports of infants with a birth weight less than or equal to $300 \mathrm{~g}$. At the same time, this is one of the more complete descriptions of the respiratory and gastrointestinal clinical courses and management of these infants in the NICU. Nevertheless, further reports on the clinical courses and management of similar infants will be invaluable.

\section{Conclusion}

Ensuring the survival of infants weighing less than or equal to $300 \mathrm{~g}$ is challenging. As their lungs and digestive tract are tiny and fragile, they require more sophisticated and delicate management than do infants weighing greater than $300 \mathrm{~g}$. However, our experience in managing this infant with a birth weight of $258 \mathrm{~g}$ shows the importance of combining traditional and modern management methods to ensure survival. When managing infants weighing less than or equal to $300 \mathrm{~g}$, it is also important to consider their special difficulties and apply management techniques familiar to each unit. We strongly hope that our experience will enable others to save more infants weighing less than or equal to $300 \mathrm{~g}$ and improve their long-term prognosis.

\section{Conflict of Interest}

None declared.

\section{Acknowledgments}

The authors wish to acknowledge all the staff members in the Division of Neonatology at Nagano Children's Hospital for their contribution to the clinical management, especially MW Makiko Noda as a primary nurse. We thank the infant, his parents, and his siblings for their contribution. We would like to thank Editage (www.editage.com) for English language editing. No funding was received for this study.

\section{References}

1 Lau C, Ambalavanan N, Chakraborty H, Wingate MS, Carlo WA. Extremely low birth weight and infant mortality rates in the United States. Pediatrics 2013;131(05):855-860

2 Itabashi K, Horiuchi T, Kusuda S, et al. Mortality rates for extremely low birth weight infants born in Japan in 2005. Pediatrics 2009; 123(02):445-450

3 Inoue H, Ochiai M, Yasuoka K, et al; Neonatal Research Network of Japan (NRNJ) Early mortality and morbidity in infants with birth weight of 500 grams or less in Japan.J Pediatr 2017;190:112-117.e3

4 Griffin IJ, Lee HC, Profit J, Tancedi DJ. The smallest of the small: short-term outcomes of profoundly growth restricted and profoundly low birth weight preterm infants. J Perinatol 2015;35 (07):503-510

5 Salihu HM, Emusu D, Aliyu ZY, Kirby RS, Alexander GR. Survival of "pre-viable" infants in the United States. Wien Klin Wochenschr 2005;117(9-10):324-332

6 Sauve RS, Robertson C, Etches P, Byrne PJ, Dayer-Zamora V. Before viability: a geographically based outcome study of infants weighing 500 grams or less at birth. Pediatrics 1998;101(3, Pt 1):438-445

7 Gillone J, Banait N, Miller N, Ward Platt M, Harigopal S. Outcomes of infants with a birthweight less than or equal to $500 \mathrm{~g}$ in Northern England: 15 years experience. Acta Paediatr 2018;107 (02):223-226

8 Brumbaugh JE, Hansen NI, Bell EF, et al; National Institute of Child Health and Human Development Neonatal Research Network. Outcomes of extremely preterm infants with birth weight less than 400 g. JAMA Pediatr 2019;173(05):434-445

9 The University of Iowa. The Tiniest Babies. Accessed 9 October 2021 at: https://webapps1.healthcare.uiowa.edu/TiniestBabies/index.aspx

10 Inoue $\mathrm{H}$, Ochiai $\mathrm{M}$, Sakai $\mathrm{Y}$, et al; Neonatal Research Network of Japan. Neurodevelopmental outcomes in infants with birth weight $\leq 500 \mathrm{~g}$ at 3 years of age. Pediatrics 2018;142(06):e20174286

11 Muraskas JK, Rau BJ, Castillo PR, Gianopoulos J, Boyd LAC. Longterm follow-up of 2 newborns with a combined birth weight of 540 grams. Pediatrics 2012;129(01):e174-e178

12 Jobe AH, Bancalari E. Bronchopulmonary dysplasia. Am J Respir Crit Care Med 2001;163(07):1723-1729

13 Muraskas JK, Myers TF, Lambert GH, Anderson CL. Intact survival of a 280-g infant: an extreme case of growth retardation with normal cognitive development at two years of age. Acta Paediatr Suppl 1992;382:16-20

14 Hokuto I, Ikeda K, Tokieda K, Mori K, Sueoka K. An ultra premature baby of $290 \mathrm{~g}$ birth weight needed more than $500 \mathrm{mg} / \mathrm{kg}$ of calcium and phosphorus daily. Eur J Pediatr 2001;160(07):450-451

15 Kohelet D, Arbel E, Shochat R, Tavori I. Survival of a 300-g infant ventilated by high-frequency oscillatory ventilation for respiratory distress syndrome. Crit Care Med 2002;30(05):1152-1155

16 Arimitsu T, Wakabayashi D, Tamaoka S, Takahashi M, Hida M, Takahashi T. Case report: intact survival of a marginally viable male infant born weighing 268 grams at 24 weeks gestation. Front Pediatr 2021;8:628362 
17 Rehan VK, Fong J, Lee R, et al. Mechanism of reduced lung injury by high-frequency nasal ventilation in a preterm lamb model of neonatal chronic lung disease. Pediatr Res 2011;70(05):462-466

18 Zivanovic S, Peacock J, Alcazar-Paris M, et al. Late outcomes of a randomized trial of high-frequency oscillation in neonates. $\mathrm{N}$ Engl J Med 2014;370(12):1121-1130

19 Cools F, Offringa M, Askie LM, Askie LM. Elective high frequency oscillatory ventilation versus conventional ventilation for acute pulmonary dysfunction in preterm infants. Cochrane Database Syst Rev 2015;19(03):CD000104

20 Iscan B, Duman N, Tuzun F, Kumral A, Ozkan H. Impact of volume guarantee on high-frequency oscillatory ventilation in preterm infants: a randomized crossover clinical trial. Neonatology 2015; 108(04):277-282

21 Enomoto M, Keszler M, Sakuma M, et al. Effect of volume guarantee in preterm infants on high-frequency oscillatory ventilation: a pilot study. Am J Perinatol 2017;34(01):26-30

22 Belteki G, Morley CJ. High-frequency oscillatory ventilation with volume guarantee: a single-centre experience. Arch Dis Child Fetal Neonatal Ed 2019;104(04):F384-F389

23 Tuzun F, Deliloglu B, Cengiz MM, Iscan B, Duman N, Ozkan H. Volume guarantee high-frequency oscillatory ventilation in preterm infants with RDS: tidal volume and $\mathrm{DCO}_{2}$ levels for optimal ventilation using open-lung strategies. Front Pediatr 2020;8:105

24 Lee BK, Shin SH, Jung YH, Kim EK, Kim HS. Comparison of NIVNAVA and NCPAP in facilitating extubation for very preterm infants. BMC Pediatr 2019;19(01):298

25 Yagui AC, Gonçalves PA, Murakami SH, Santos AZ, Zacharias RSB, Rebello $\mathrm{CM}$. Is noninvasive neurally adjusted ventilatory assistance (NIV-NAVA) an alternative to NCPAP in preventing extubation failure in preterm infants? J Matern Fetal Neonatal Med 2021; 34(22):3756-3760

26 Kallio M, Mahlman M, Koskela U, et al. NIV NAVA versus nasal CPAP in premature infants: a randomized clinical trial. Neonatology 2019;116(04):380-384

27 Krasna IH, Rosenfeld D, Salerno P. Is it necrotizing enterocolitis, microcolon of prematurity, or delayed meconium plug? A dilemma in the tiny premature infant. J Pediatr Surg 1996;31(06):855-858

28 Dimmitt RA, Moss RL. Meconium diseases in infants with very low birth weight. Semin Pediatr Surg 2000;9(02):79-83

29 Emil S, Nguyen T, Sills J, Padilla G. Meconium obstruction in extremely low-birth-weight neonates: guidelines for diagnosis and management. J Pediatr Surg 2004;39(05):731-737

30 Shinohara T, Tsuda M, Koyama N. Management of meconiumrelated ileus in very low-birthweight infants. Pediatr Int 2007;49 (05):641-644
31 Ein SH, Shandling B, Reilly BJ, Stephens CA. Bowel perforation with nonoperative treatment of meconium ileus. J Pediatr Surg 1987; 22(02):146-147

32 Michikata K, Kodama Y, Kaneko M, et al. Oral diatrizoate acid for meconium-related ileus in extremely preterm infants. Pediatr Int (Roma) 2018;60(08):714-718

33 Farhath S, He Z, Nakhla T, et al. Pepsin, a marker of gastric contents, is increased in tracheal aspirates from preterm infants who develop bronchopulmonary dysplasia. Pediatrics 2008;121 (02):e253-e259

34 Misra S, Macwan K, Albert V. Transpyloric feeding in gastroesophageal-reflux-associated apnea in premature infants. Acta Paediatr 2007;96(10):1426-1429

35 Malcolm WF, Smith PB, Mears S, Goldberg RN, Cotten CM. Transpyloric tube feeding in very low birthweight infants with suspected gastroesophageal reflux: impact on apnea and bradycardia. J Perinatol 2009;29(05):372-375

36 Watson J, McGuire W. Transpyloric versus gastric tube feeding for preterm infants. Cochrane Database Syst Rev 2013;2013(02): CD003487

37 Ehrenkranz RA, Dusick AM, Vohr BR, Wright LL, Wrage LA, Poole WK. Growth in the neonatal intensive care unit influences neurodevelopmental and growth outcomes of extremely low birth weight infants. Pediatrics 2006;117(04):1253-1261

38 Franz AR, Pohlandt F, Bode H, et al. Intrauterine, early neonatal, and postdischarge growth and neurodevelopmental outcome at 5.4 years in extremely preterm infants after intensive neonatal nutritional support. Pediatrics 2009;123(01): e101-e109

39 Belfort MB, Rifas-Shiman SL, Sullivan T, et al. Infant growth before and after term: effects on neurodevelopment in preterm infants. Pediatrics 2011;128(04):e899-e906

40 Zozaya C, Díaz C, Saenz de Pipaón M. How should we define postnatal growth restriction in preterm infants? Neonatology 2018;114(02):177-180

41 Arslanoglu S, Boquien CY, King C, et al. Fortification of human milk for preterm infants: Update and recommendations of the European milk bank association (EMBA) working group on human milk fortification. Front Pediatr 2019;7:76

42 Vaidya UV, Hegde VM, Bhave SA, Pandit AN. Vegetable oil fortified feeds in the nutrition of very low birthweight babies. Indian Pediatr 1992;29(12):1519-1527

43 Kwon S-J, Park H-K, Kim M-S. Nutritional management for intolerance to human milk fortifier in a preterm small-for-gestational-age infant: a case report. Clin Nutr Res 2020;9(03): $235-240$ 